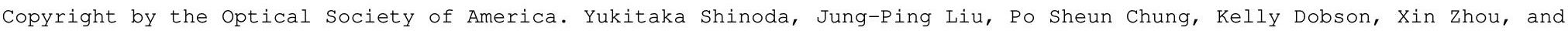
Ting-Chung Poon, "Three-dimensional complex image coding using a circular Dammann grating," Appl. Opt. 50, B38-B45 (2011); doi: 10.1364/ao.50.000b38

\title{
Three-dimensional complex image coding using a circular Dammann grating
}

\author{
Yukitaka Shinoda, ${ }^{1}$ Jung-Ping Liu, ${ }^{2, *}$ Po Sheun Chung, ${ }^{3}$ Kelly Dobson, ${ }^{4}$ \\ Xin Zhou, ${ }^{5}$ and Ting-Chung Poon ${ }^{4}$ \\ 'Department of Electrical Engineering, Nihon University, Tokyo, 101-8308, Japan \\ ${ }^{2}$ Department of Photonics, Feng Chia University, 100 Wenhwa Road, Seatwen, \\ Taichung, 40724 Taiwan \\ ${ }^{3}$ Department of Electronic Engineering, City University of Hong Kong, \\ 83, Tat Chee Avenue, Kowloon Tong, Hong Kong, China \\ ${ }^{4}$ Bradley Department of Electrical and Computer Engineering, Virginia Tech, \\ Blacksburg, Virginia 24061, USA \\ ${ }^{5}$ Department of Opto-Electronics Science and Technology, Sichuan University, \\ Chengdu 610065, China \\ ${ }^{*}$ Corresponding author: jpliu@fcu.edu.tw
}

Received 30 June 2010; revised 19 October 2010; accepted 16 November 2010; posted 16 November 2010 (Doc. ID 130888); published 16 December 2010

\begin{abstract}
Recently, optical image coding using a circular Dammann grating (CDG) has been proposed and investigated. However, the proposed technique is intensity based and could not be used for three-dimensional (3D) image coding. In this paper, we investigate an optical image coding technique that is complexamplitude based. The system can be used for $3 \mathrm{D}$ image coding. The complex-amplitude coding is provided by a circular Dammann grating through the use of a digital holographic recording technique called optical scanning holography. To decode the image, along the depth we record a series of pinhole holograms coded by the CDG. The decoded reconstruction of each depth location is extracted by the measured pinhole hologram matched to the desired depth. Computer simulations as well as experimental results are provided. (C) 2010 Optical Society of America
\end{abstract}

OCIS codes: $\quad 090.1995,100.1160,100.3010,100.6890$.

\section{Introduction}

Optical image coding is an important area of research, as it can be applied to optical encryption. Optical image coding is often a logical choice as optical images needing to be coded are in the optical domain. In addition, optical image coding, as opposed to electronic or digital image coding, can provide many degrees of freedom for coding. Recently, there has been a review article on the subject [1]. In this paper, we investigate a 3D optical image coding technique that is complex-amplitude based. The technique is based

0003-6935/11/070B38-08 $\$ 15.00 / 0$

(C) 2011 Optical Society of America on the use of a digital holographic recording called optical scanning holography (OSH) pioneered by Poon [2]. The OSH technique is based on a two-pupil interferometric scanning system. Because the pupils can be designed arbitrarily, the OSH system has achieved a variety of applications, such as incoherent image processing, optical recognition of 3D objects, holographic microscopy, and optical remote sensing [2]. We propose to perform 3D optical image encryption with a circular Dammann grating (CDG) through the use of OSH. In contrast to a recent intensity-based optical system based on the grating for two-dimensional (2D) image coding [3], the present coding method is complex-amplitude based 
and is suitable for 3D image coding. Because the Dammann grating can be easily fabricated and duplicated, decryption will be easier than those encoded with a random phase mask [ $\underline{4}, \underline{5}]$. In Section 2 , we provide a brief introduction to OSH and summarize some of the important results pertinent to our present investigation of optical encryption. In Section 3 , we discuss the theory on complex optical coding. We provide some computer simulations in Section 4. In Section $\underline{5}$, we discuss optical results and compare them to those shown in Section 4. Finally, in Section $\underline{6}$, we make some concluding remarks.

\section{Optical Scanning Holography}

The schematic setup of an OSH system is shown in Fig. 1. One collimated laser beam with angular frequency of $\omega_{0}$ and another collimated laser beam with angular frequency of $\omega_{0}+\Omega$, respectively, pass through pupils $p_{1}(x, y)$ and $p_{2}(x, y)$. The two beams are then combined by a beam splitter (BS). The combined laser beam passes through a positive lens L1 and is used to scan the object specified by the intensity transmittance of $T\left(x, y ; z_{0}\right)$, located $z=z_{0}$ away from the focal plane of lens L1. The light scattered from the object is then collected by another positive lens L2 and measured by a photodetector (PD). The photodetector delivers a heterodyne current $i_{\Omega}(x, y, t)$ at angular frequency $\Omega$ for immediate demodulation, as shown in Fig. 1.

For demodulation, it is basically a lock-in amplifier (electronic multiplier plus a low-pass filter) giving the in-phase current $i_{c}(x, y)$ and the quadrature current $i_{s}(x, y)$ as outputs:

$$
\begin{aligned}
& i_{c}=\operatorname{Re}\left\{i_{\Omega p}(x, y)\right\}, \\
& i_{s}=\operatorname{Im}\left\{i_{\Omega p}(x, y)\right\},
\end{aligned}
$$

where $i_{\Omega}(x, y, t)=i_{\Omega p}(x, y) \exp (j \Omega t)$ and $\operatorname{Re}\{$.$\} and$ $\operatorname{Im}\{$.$\} denote the operations of taking the real part$ and the imaginary part, respectively. It is noted that the information of the scanned and processed version of $T\left(x, y ; z_{0}\right)$, using Eqs. (1) and (2), is included in the complex output $i_{\Omega p}(x, y)$ as [ $[6]$

$$
\begin{aligned}
i_{\Omega p}(x, y) & =i_{c}+j i_{s}=F^{-1}\left\{F\left\{T\left(x, y ; z_{0}\right)\right\} \times \operatorname{OTF}\left(k_{x}, k_{y} ; z_{0}\right)\right\} \\
& =T\left(x, y ; z_{0}\right) \otimes F^{-1}\left\{\operatorname{OTF}\left(k_{x}, k_{y} ; z_{0}\right)\right\},
\end{aligned}
$$

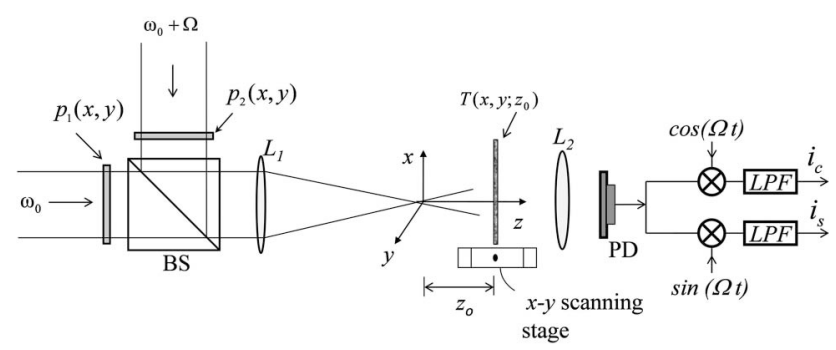

Fig. 1. Schematic setup of an OSH system: $\otimes$, electronic multipliers; and LPF, low-pass filter. where $F\{$.$\} and F^{-1}\{$.$\} denote the Fourier transform$ and the inverse Fourier transform, respectively; $F^{-1}\left\{\mathrm{OTF}\left(k_{x}, k_{y} ; z_{0}\right)\right\}$ can be recognized as the impulse response of the system that can be measured by simply putting a pinhole as the object, i.e., let $T\left(x, y ; z_{0}\right)=\delta(x, y)$ at the location $z=z_{0}$. We will discuss this important measurement in a subsequent section. Finally, $\otimes$ denotes the operation of the $2 \mathrm{D}$ convolution, and OTF stands for the optical transfer function of the system at $z$, which has been shown to be expressed as [7]

$$
\begin{aligned}
\operatorname{OTF}\left(k_{x}, k_{y} ; z\right)= & \exp \left[j \frac{z}{2 k_{0}}\left(k_{x}^{2}+k_{y}^{2}\right)\right] \\
& \times \iint p_{1}^{*}\left(x^{\prime}, y^{\prime}\right) p_{2}\left(x^{\prime}+\frac{f}{k_{0}} k_{x}, y^{\prime}+\frac{f}{k_{0}} k_{y}\right) \\
& \times \exp \left[j \frac{z}{f}\left(x^{\prime} k_{x}+y^{\prime} k_{y}\right)\right] d x^{\prime} d y^{\prime}
\end{aligned}
$$

where $k_{x}$ and $k_{y}$ are spatial radian frequencies, $k_{0}=$ $2 \pi / \lambda$ is the wavenumber, and $\lambda$ is the wavelength of the laser used. From Eq. (3), we can envision that the object spectrum, i.e., $\left.\bar{F} \bar{T}\left(x, y ; z_{0}\right)\right\}$, is coded by $\operatorname{OTF}\left(k_{x}, k_{y} ; z_{0}\right)$. Note that the OTF can be modified by using different pupil functions so that the pupils can be regarded as a freedom of the system. In typical holographic imaging, often referred to as OSH, the pupils are set as $p_{1}(x, y)=1$ and $p_{2}(x, y)=\delta(x, y)$ so that Eq. ( $\underline{4})$ is reduced to

$$
\begin{aligned}
\operatorname{OTF}\left(k_{x}, k_{y} ; z\right) & =\exp \left[-j \frac{z}{2 k_{0}}\left(k_{x}^{2}+k_{y}^{2}\right)\right] \\
& =\mathrm{OTF}_{\mathrm{OSH}}\left(k_{x}, k_{y} ; z\right) .
\end{aligned}
$$

Hence, for simply holographic recording, the coding OTF is simply given by Eq. (5), which turns out to be the transfer function in free space under the paraxial approximation [6]. Thus, the object function $T\left(x, y ; z_{0}\right)$, using Eq. (3), can be retrieved easily by the following calculation:

$$
\begin{aligned}
i_{\Omega p}(x, y) \otimes & F^{-1}\left\{\mathrm{OTF}_{\mathrm{OSH}}^{*}\left(f_{x}, f_{y} ; z_{0}\right)\right\} \\
= & F^{-1}\left\{F\left\{T\left(x, y ; z_{0}\right)\right\} \times \mathrm{OTF}_{\mathrm{OSH}}\left(k_{x}, k_{y} ; z_{0}\right)\right. \\
& \left.\times \mathrm{OTF}_{\mathrm{OSH}}^{*}\left(k_{x}, k_{y} ; z_{0}\right)\right\} \\
= & T\left(x, y ; z_{0}\right),
\end{aligned}
$$

because

$$
\left.\mathrm{OTF}_{\mathrm{OSH}}\left(k_{x}, k_{y} ; z\right) \times \mathrm{OTF}_{\mathrm{OSH}}^{*}\left(k_{x}, k_{y} ; z\right)\right\}=1,
$$

for any $z$. In fact, $i_{\Omega p}(x, y)$, given by Eq. (3) with Eq. (5) as the coding OTF, is the complex digital hologram of $T\left(x, y ; z_{0}\right)$. From Eq. (6a), we recognize that the coding OTF, OTF $\mathrm{OTF}_{\mathrm{OSH}}\left(k_{x}, k_{y} ; z_{0}\right)$ for simply holographic recording and the decoding $\mathrm{OTF}$, $\mathrm{OTF}_{\text {decoding }}\left(k_{x}, k_{y} ; z\right)$, is $\mathrm{OTF}_{\mathrm{OSH}}^{*}\left(k_{x}, k_{y} ; z_{0}\right)$ for holographic reconstruction. 


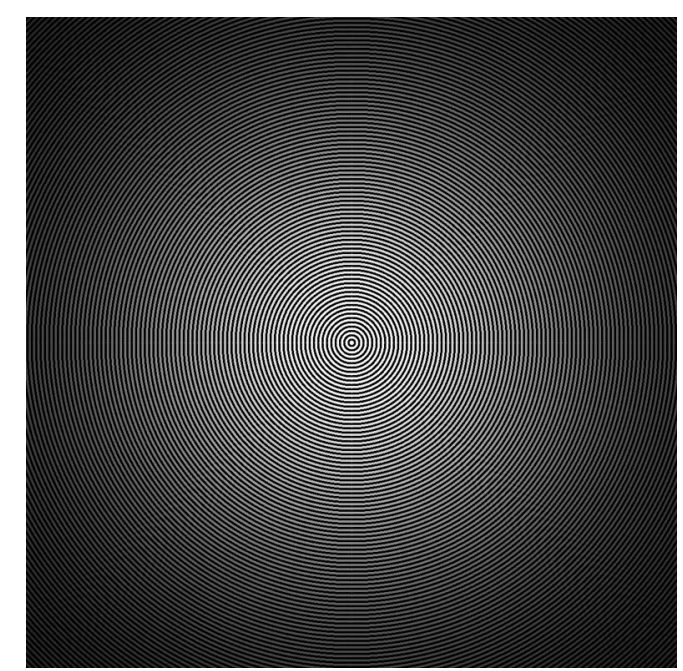

Fig. 2. Real part of the optical field just behind the Dammann grating.

When Eq. (6b) is satisfied, we have reconstructed holographically the original image.

In the above analysis, only a planar intensity transmittance in a 3D space is considered. A real $3 \mathrm{D}$ object can be regarded as a combination of multiple $2 \mathrm{D}$ slices at various axial depths. Assume that if there are a total of $M$ object slices, the complex output $i_{\Omega p}(x, y)$ should be expressed as

$i_{\Omega p}(x, y)=\sum_{m=1}^{M} T_{m}\left(x, y ; z_{m}\right) \otimes F^{-1}\left\{\mathrm{OTF}\left(k_{x}, k_{y} ; z_{m}\right)\right\},(7)$

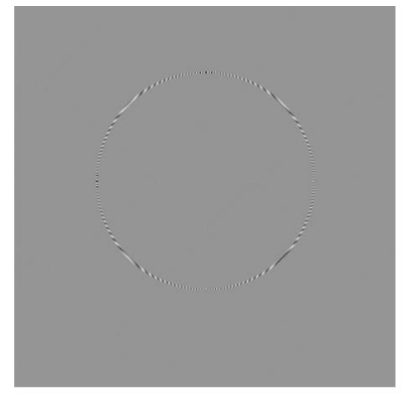

(a)

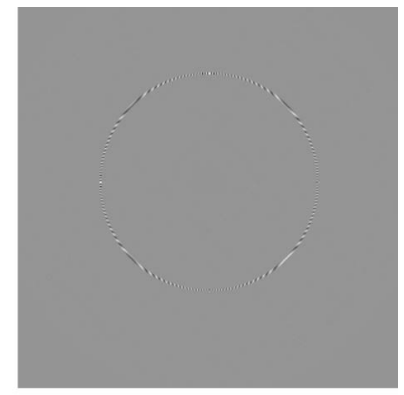

(b)

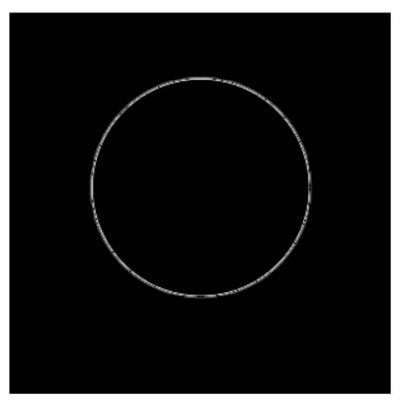

(c) where the subscript $m$ stands for the $m$ th object slice located at $z=z_{0}$. Therefore, we can only retrieve a single slice of the object by Eq. (6), while other slices will contribute to defocus haze. The method of removing the defocus haze in holography, i.e., sectioning, is beyond the scope of the paper and can be found in Refs. [요미].

\section{Complex Optical Coding}

In Section 2, we discussed the coding and decoding OTFs for a simple holographic recording. In this section, we discuss the coding and decoding OTFs for use in complex optical image coding. In general, Eq. (6a) is given by a general OTF given by Eqs. ()ㅜ and $(\underline{6 a})$ becomes

$$
\begin{aligned}
i_{\Omega p}(x, y) \otimes & F^{-1}\left\{\operatorname{OTF}^{*}\left(k_{x}, k_{y} ; z_{0}\right)\right\} \\
= & F^{-1}\left\{F\left\{T\left(x, y ; z_{0}\right)\right\} \times \operatorname{OTF}\left(k_{x}, k_{y} ; z_{0}\right)\right. \\
& \left.\times \operatorname{OTF}^{*}\left(k_{x}, k_{y} ; z_{0}\right)\right\} .
\end{aligned}
$$

In this general case, we have

$$
\begin{gathered}
\operatorname{OTF}_{\text {coding }}\left(k_{x}, k_{y} ; z_{0}\right)=\operatorname{OTF}\left(k_{x}, k_{y} ; z_{0}\right), \\
\operatorname{OTF}_{\text {decoding }}\left(k_{x}, k_{y} ; z_{0}\right)=\operatorname{OTF}^{*}\left(k_{x}, k_{y} ; z_{0}\right),
\end{gathered}
$$

i.e., the coding OTF and the decoding OTF are related by a simple complex conjugation.

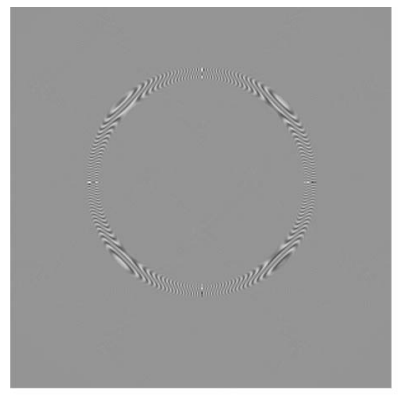

(a)

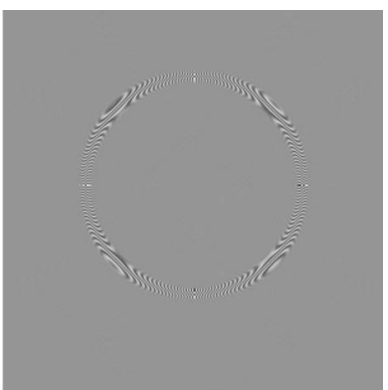

(b)

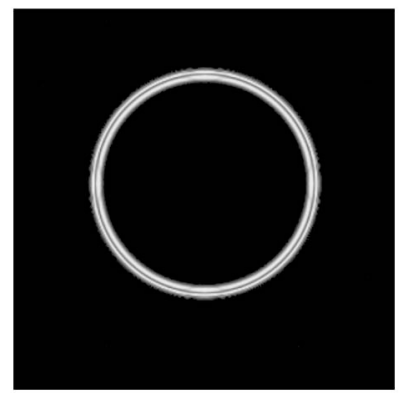

(c)

Fig. 4. (a) Real part, (b) imaginary part, and (c) absolute value of the diffraction pattern at the plane $z_{0}=4.5 \mathrm{~cm}$.
Fig. 3. (a) Real part, (b) imaginary part, and (c) absolute value of the diffraction pattern at the back focal plane of L1 $\left(z_{0}=0\right)$. 


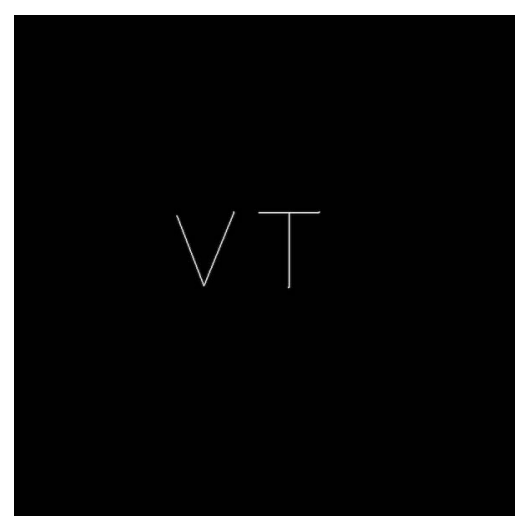

Fig. 5. Object to be coded.

When

$$
\begin{aligned}
\mathrm{OTF}_{\text {coding }} \times \mathrm{OTF}_{\text {decoding }}= & \operatorname{OTF}\left(k_{x}, k_{y} ; z_{0}\right) \\
& \left.\times \operatorname{OTF}^{*}\left(k_{x}, k_{y} ; z_{0}\right)\right\}=1,(10 \mathrm{a})
\end{aligned}
$$

or

$$
\begin{aligned}
F^{-1}\left\{\mathrm{OTF}\left(k_{x}, k_{y} ; z_{0}\right)\right\} & \otimes F^{-1}\left\{\operatorname{OTF}^{*}\left(k_{x}, k_{y} ; z_{0}\right)\right\} \\
& =\delta(x, y),
\end{aligned}
$$

the original image can be retrieved or decoded faithfully. However, from Eq. (4), we see that Eq. (10) is, in general, not necessarily satisfied, depending on the choices of the two pupils. We shall now consider very specific pairs of the pupils that will satisfy Eq. (10). We assume that the first pupil function $p_{1}(x, y)$ is specified while the second pupil $p_{2}(x, y)$ is a delta function. Thus Eq. (4) becomes

$$
\begin{aligned}
\mathrm{OTF}_{\text {coding }}\left(k_{x}, k_{y} ; z\right)= & \exp \left[-j \frac{z}{2 k_{0}}\left(k_{x}^{2}+k_{y}^{2}\right)\right] \\
& \times p_{1}^{*}\left(-\frac{f}{k_{0}} k_{x},-\frac{f}{k_{0}} k_{y}\right) \\
= & \mathrm{OTF}_{\mathrm{OSH}}\left(k_{x}, k_{y} ; z\right) \\
& \times p_{1}^{*}\left(-\frac{f}{k_{0}} k_{x},-\frac{f}{k_{0}} k_{y}\right) .
\end{aligned}
$$

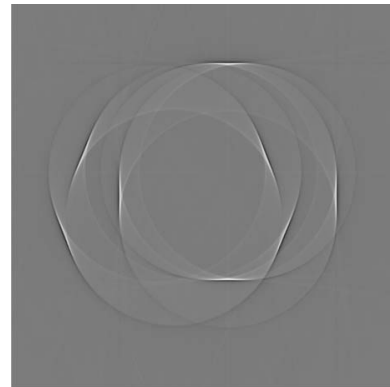

(a)

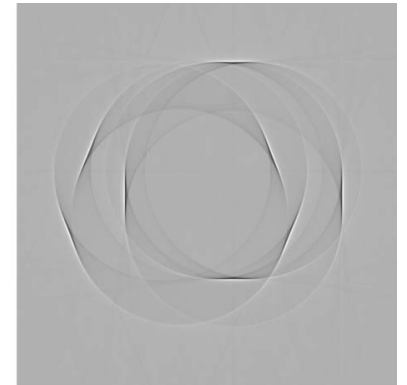

(b)
Fig. 6. (a) Real part and (b) imaginary part of the coded complex hologram for the object located at the back focal plane of L1 $\left(z_{0}=0\right)$.

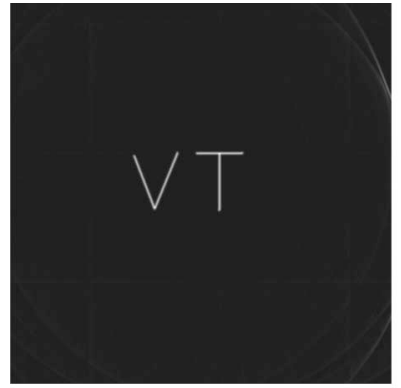

(a)

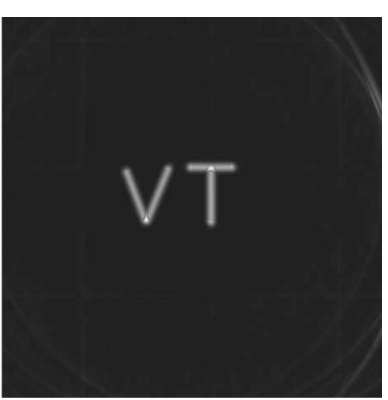

(b)
Fig. 7. Images reconstructed at (a) $z_{0}=0$ and (b) $z_{0}=4.5 \mathrm{~cm}$ while the object is located at $z_{0}=0$.

From the above equation, we note that the first product is the OTF for holographic recording, i.e., $\mathrm{OTF}_{\mathrm{OSH}}\left(k_{x}, k_{y} ; z\right)$ from Eq. (5). We can therefore consider the second product as a mechanism for coding the object spectrum before holographic recording. In other words, when $p_{1}=1$, we simply have holographic coding, i.e., holographic recording. However, in general, the object information is coded by the pupil function $p_{1}(x, y)$, and we call $p_{1}(x, y)$ a coding pupil. A merit of optical coding is that the object information can be disturbed optically so that it cannot be easily identified without the decoding process - a notion of encryption and decryption. For an arbitrary coding pupil, to decode the complex hologram based on Eq. (9), we have

$$
\begin{aligned}
& \mathrm{OTF}_{\text {coding }}\left(k_{x}, k_{y} ; z\right) \times \mathrm{OTF}_{\text {decoding }}\left(k_{x}, k_{y} ; z\right) \\
& =\left(\mathrm{OTF}_{\mathrm{OSH}} \times p_{1}^{*}\right) \times\left(\mathrm{OTF}_{\mathrm{OSH}}^{*} \times p_{1}\right)=p_{1}^{*} \times p_{1},
\end{aligned}
$$

which is not unity in general for a given $p_{1}(x, y)$ and that the object information cannot be decoded directly using $\mathrm{OTF}_{\text {decoding }}\left(k_{x}, k_{y} ; z\right)$ through the application of Eq. (10). Under such a situation, decoding of the object can be always found by inverse filtering:

$$
\operatorname{OTF}_{\text {decoding }}\left(k_{x}, k_{y} ; z\right)=\frac{1}{\operatorname{OTF}_{\text {coding }}\left(k_{x}, k_{y} ; z\right)+\varepsilon},
$$

provided $\mathrm{OTF}_{\text {coding }}\left(k_{x}, k_{y} ; z\right)$ is known, where $\varepsilon$ is a small number added manually to avoid the diver-

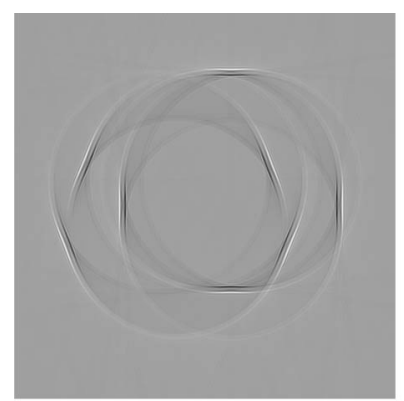

(a)

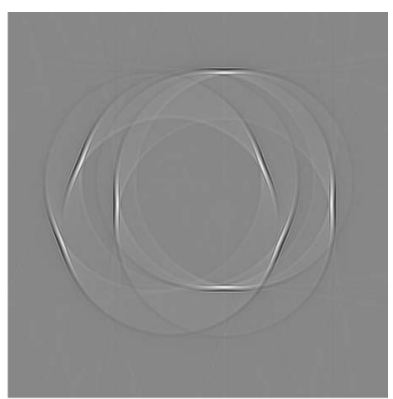

(b)
Fig. 8. (a) Real part and (b) imaginary part of the hologram for the object located at $z_{0}=4.5 \mathrm{~cm}$. 


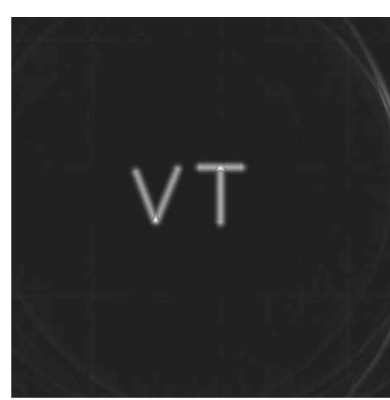

(a)

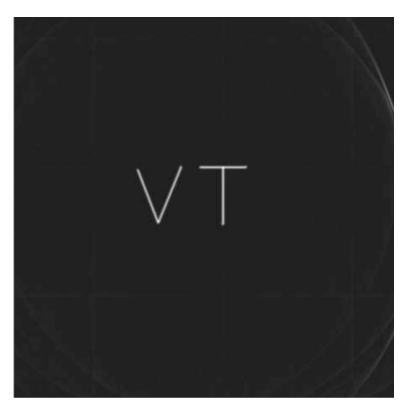

(b)

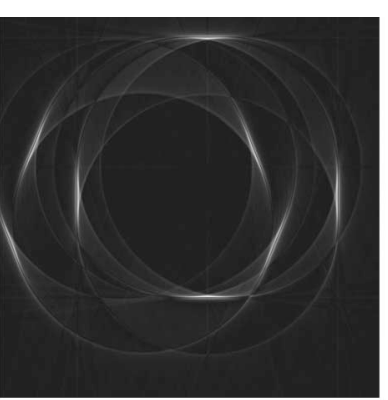

(a)

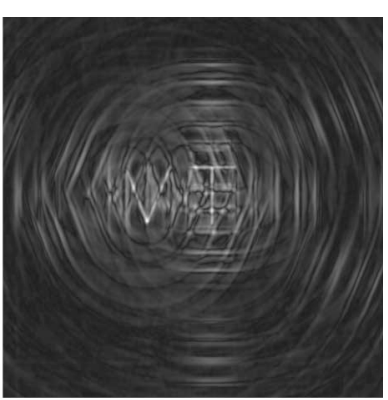

(b)

Fig. 9. Images reconstructed at (a) $z_{0}=0$ and (b) $z_{0}=4.5 \mathrm{~cm}$ while the object is located at $z_{0}=4.5 \mathrm{~cm}$.

gence at $\operatorname{OTF}_{\text {coding }}\left(k_{x}, k_{y} ; z\right)=0$. As a result, the decoding function exhibits the property

$$
\operatorname{OTF}_{\text {encoding }}\left(k_{x}, k_{y} ; z\right) \times \operatorname{OTF}_{\text {decoding }}\left(k_{x}, k_{y} ; z\right) \approx 1 \text {. }
$$

The inverse filtering method is, however, not convenient because it depends on the reconstructed distance $z$. Thus we need multiple decoding functions if the object target is 3D. Moreover, inverse filtering will induce additionally high spatial-frequency noise in the reconstructed image. However, if $p_{1}(x, y)$ is chosen to be a phase function, the below condition can always be met:

$$
\begin{aligned}
\mathrm{OTF}_{\text {coding }}\left(k_{x}, k_{y} ; z\right) \times \mathrm{OTF}_{\text {decoding }}\left(k_{x}, k_{y} ; z\right) & =p_{1}^{*} \times p_{1} \\
& =1
\end{aligned}
$$

The above condition is one of the main results with the OSH system for complex coding and decoding applications. In this paper we use a CDG as the pupil function $p_{1}(x, y)$. A CDG is a binary phase grating $(0$ and $\pi$ ) with circular symmetry [12]. The grating has been used for a variety of applications, such as in angle measurement and, most recently, in area mea-

Fig. 10. (a) Using Fresnel diffraction and (b) applying a decoding Dammann grating of period $100 \mu \mathrm{m}$ to reconstruct the coded complex hologram shown in Fig. $\underline{8}$. The reconstruction distance is at $z_{0}=4.5 \mathrm{~cm}$.

surement $[13,14]$. Because an ideal CDG is a purely phase object, it is apparent from Eq. (11) that the coding OTF involving a Dammann grating will satisfy Eq. (15). In any case, for decoding, we need to know (through measurement) the coding OTF, which can be done by placing a pinhole as an image to measure the impulse response of the system, and the Fourier transform of the impulse response will give the coding OTF.

\section{Coding and Decoding by a Circular Dammann Grating as the Coding Pupil Function: Simulations}

In the simulation, we use a Gaussian beam with a waist of $8 \mathrm{~mm}$ and wavelength of $0.6328 \mu \mathrm{m}$ to illuminate the Dammann grating. Figure 2 shows the optical field (only the real part of the complex field is shown) just behind the Dammann grating. Ignoring some of the aliasing effect, we basically notice the circular pattern of the field distribution. The period of the Dammann grating is $80 \mu \mathrm{m}$, and the focal length of lens L1 is $75 \mathrm{~cm}$. These are the data matched for optical experiments, to be demonstrated in Section 5 . The diffraction pattern of the CDG is a ring at the

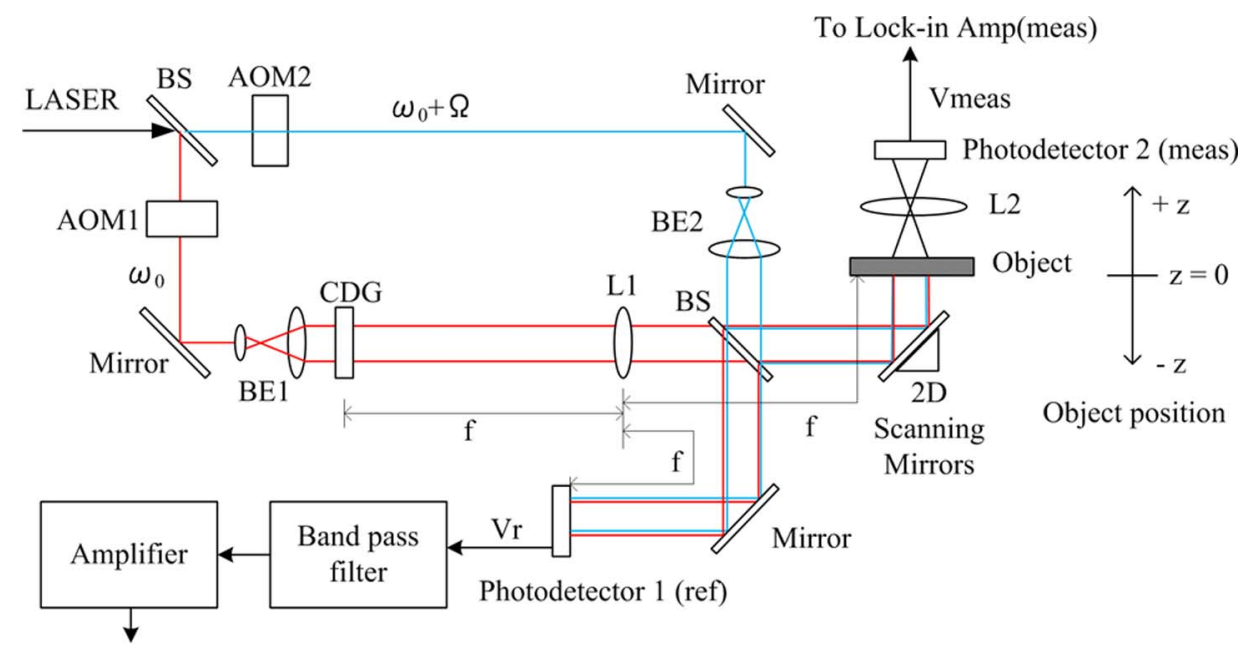

To Lock-in Amp(ref)

Fig. 11. (Color online) Optical system for coding using a CDG as the coding pupil: BE1, BE2, beam expanders; AOM1, AOM2, acousto-optic frequency shifter; BS, beam splitter. 


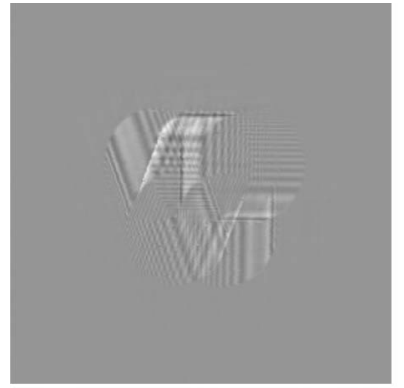

(a)

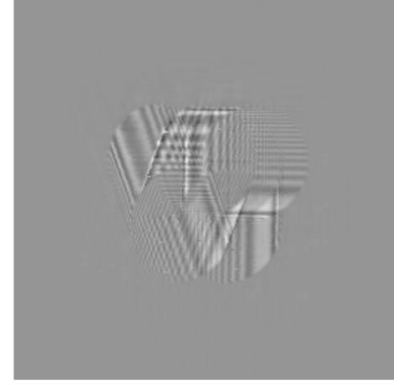

(b)

Fig. 12. (a) Real part and (b) imaginary part of a coded complex hologram, where the object "VT" is located at $z_{0}=4.5 \mathrm{~cm}$.

back focal plane of lens L1. Figures 3(a)-3(c) show the real part, imaginary part, and absolute value of the diffraction pattern at the focal plane of lens $\mathrm{L} 1$, respectively. If the observing plane deviates from the focal plane, the diffraction pattern will become a twin ring, which is shown in Fig. 4. These complex diffracted patterns, in fact, are used to code the object in a complex fashion. In producing a coded complex hologram, we set the object pattern shown in Fig. 5 at the back focal plane of L1, i.e., $z_{0}=0$. We simulate complex coding according to Eq. (3), where the OTF becomes the coding OTF given by Eq. (11) with the coding pupil, $p_{1}$, to be the Dammann grating.

The real and imaginary parts of the coded complex hologram, $i_{\Omega p}(x, y)$, are shown in Fig. $\underline{6}$, in which the patterns are seriously disturbed so that no information can be identified. To decode the coded complex hologram, we calculate Eq. () according to Eqs. (9) and (11). The corresponding decoded image is shown in Fig. 7(a). The object pattern is successfully reconstructed. We also performed decoding at another location, namely $z_{0}=4.5 \mathrm{~cm}$, i.e., we used $\mathrm{OTF}_{\text {decoding }}\left(k_{x}, k_{y} ; z_{0}=4.5 \mathrm{~cm}\right)$, while the coding

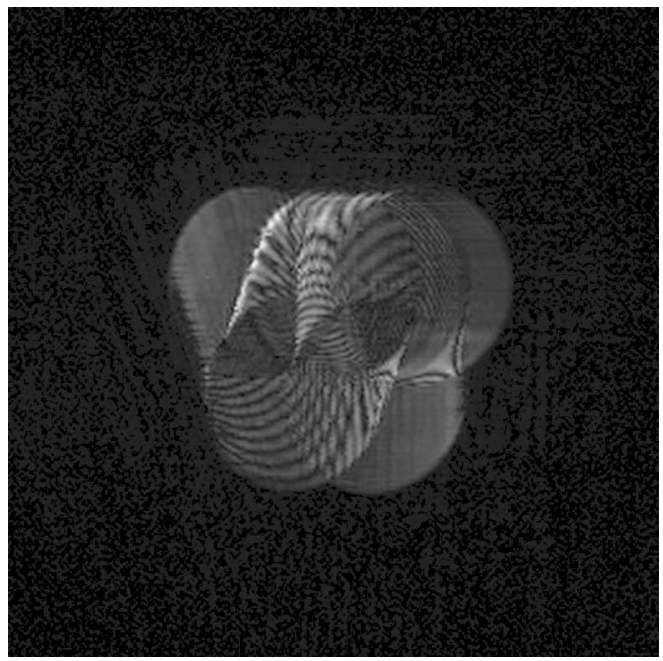

Fig. 13. Using the Fresnel diffraction to reconstruct the coded complex hologram shown in Fig. 12. The object "VT" is located at $z_{0}=4.5 \mathrm{~cm}$. The standard holographic reconstruction of the coded complex hologram is not recognizable as the hologram has been coded.

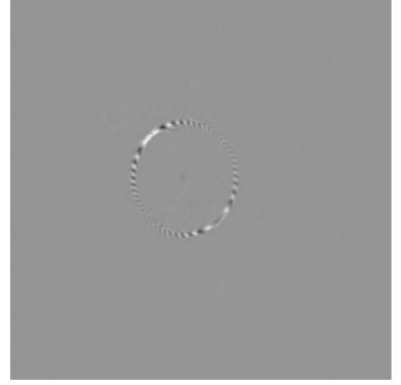

(a)

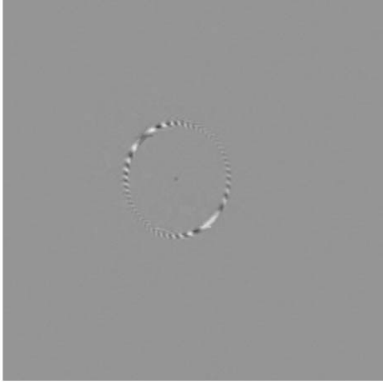

(b)
Fig. 14. Pinhole hologram measured at $z_{0}=4.5 \mathrm{~cm}$ : (a) real part of the hologram and (b) imaginary part of the hologram.

$\mathrm{OTF}$ is $\mathrm{OTF}_{\text {decoding }}\left(k_{x}, k_{y} ; z_{0}=0\right)$. The corresponding decoded image is shown in Fig. 7(b). Because the reconstruction location is not exactly at the original location of the object, even with the coding pupil correctly chosen, the reconstructed image is a little blurred, as in a general defocused imaging system. We also simulate to set the object at $z_{0}=4.5 \mathrm{~cm}$ in the coding process. The complex coded hologram is shown in Fig. 8 , with Figs. 8(a) and 8(b) being the real and imaginary part of the complex coded holograms, respectively. Figure 9(a) shows the corresponding decoded image at the reconstruction location of $z_{0}=0$, while Fig. 9(b) shows the decoded image at the reconstruction location of $z_{0}=4.5 \mathrm{~cm}$. The object pattern is also clear only at the location of the object $\left(z_{0}=4.5 \mathrm{~cm}\right)$ and is blurred elsewhere. Therefore, a $3 \mathrm{D}$ object can be coded using the CDG as the coding pupil. Note that the coded complex hologram cannot be decoded and reconstructed as a typical digital hologram. Figure 10(a) shows the reconstruction of the complex coded hologram of Fig. 8 using Fresnel diffraction at a distance of $z_{0}=4.5 \mathrm{~cm}$, which shows that the object still has been coded. In addition, in the decoding process, the decoding pupil parameter, namely the period of the CDG, should be the same as

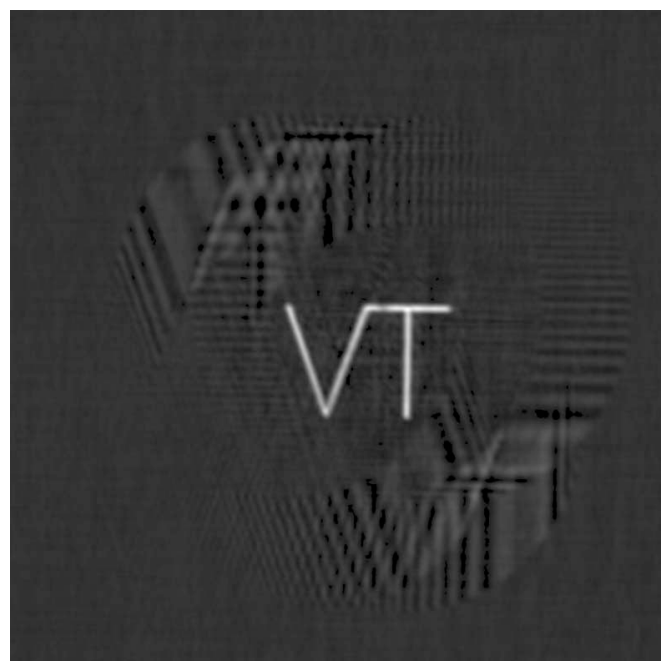

Fig. 15. Reconstruction of the complex hologram shown in Fig. 12 using the pinhole hologram shown in Fig. 14. The hologram is clearly reconstructed at the correct location of $z_{0}=4.5 \mathrm{~cm}$. 


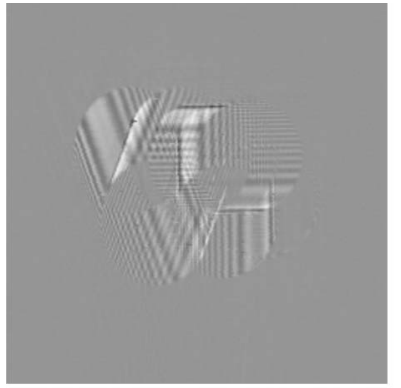

(a)

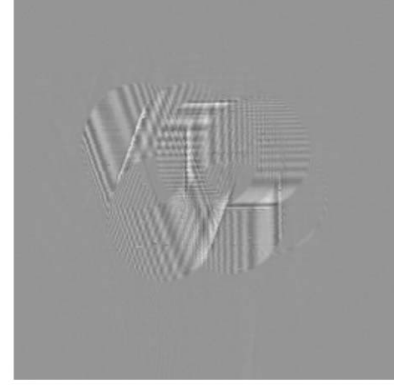

(b)

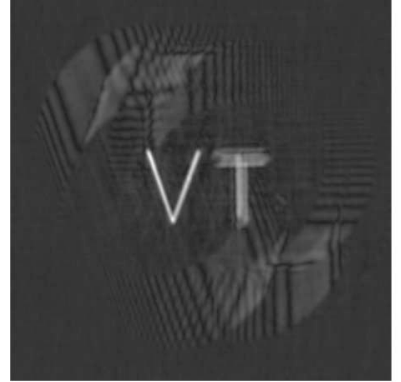

(a)

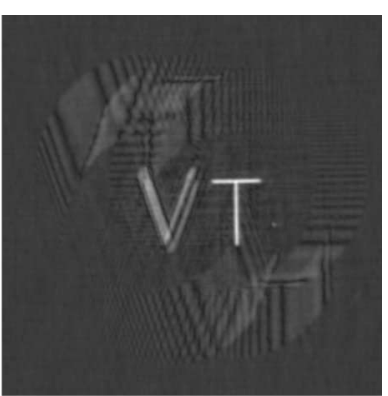

(b)

Fig. 16. Complex hologram of a $3 D$ object. "V" is located at $z_{0}=0$, and "T" is located at $z_{0}=4.5 \mathrm{~cm}$ : (a) real part of the coded complex hologram and (b) imaginary part of the coded complex hologram.

that used for coding. Figure 10(b) shows the decoding of the complex coded hologram of Fig. 8, applying a CDG of period $100 \mu \mathrm{m}$. The reconstruction is still seriously disrupted.

\section{Experimental Results}

In this section, we show some experimental results. Figure 11 shows the experimental setup. The two $\mathrm{BSs}$ and the two mirrors form the Mach-Zehnder interferometer. AOM1 and AOM2 are acousto-optic modulators upshifting the laser frequency to $\omega_{0} / 2 \pi$ and $\left(\omega_{0}+\Omega\right) / 2 \pi$, respectively, where $\Omega / 2 \pi$ is set at $50 \mathrm{kHz}$. BE1 and BE2 are the two beam expanders that provide a uniform laser beam for the circular Dammann grating (DG) and the object, respectively. The object position at $z=0$ is the focal plane of lens L1 with the focal length of $f=75 \mathrm{~cm}$. The DG is located at the front focal plane of lens L1 and is the coding pupil. The other pupil is effectively a pinhole (the focused laser spot within BE2) as the laser hitting the object is a broad laser beam. Lens L2 is simply a lens collecting the light energy onto photodetector 2 , which provides the measured electrical signal Vmeas as output. The output signal serves as an input to a lock-in amplifier (also see Fig. 1). The remaining mirror shown on the bottom of the figure is used to direct the two laser beams combined by the BS (just after lens L1) to photodetector 1 , which provides a reference heterodyne signal $\mathrm{Vr}$ at $50 \mathrm{kHz}$. The bandpass filter rejects the DC electrical signal from photodetector 1 and allows the heterodyne signal to go to the re-

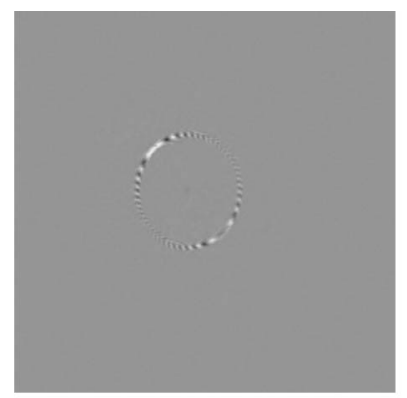

(a)

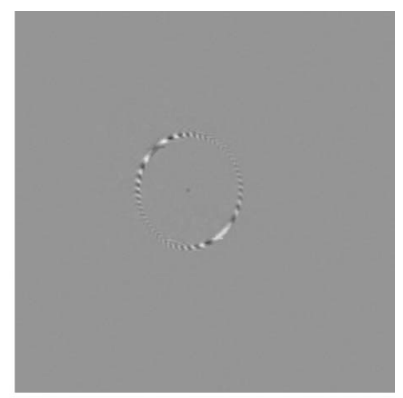

(b)

Fig. 17. Pinhole hologram measured at $z_{0}=0$ : (a) real part of the hologram and (b) imaginary part of the hologram.

Fig. 18. Reconstructions using the pinhole holograms shown in Figs. 14 and 17. (a) "V" is reconstructed correctly at $z_{0}=0$ and (b) "T" at $z_{0}=4.5 \mathrm{~cm}$.

ference input of the lock-in amplifier. This reference signal basically provides $\sin (\Omega t)$ and $\cos (\Omega t)$, as shown in Fig. 1. Figures 12(a) and 12(b) show the real and imaginary parts of a coded complex hologram, respectively, i.e., the outputs given by Eqs. (1) and (2), of the object "VT," which is located at $z_{0}=4.5 \mathrm{~cm}$. The object "VT" is approximately $1 \mathrm{in} . \times 1 \mathrm{in}$. and is transmitted on an opaque background with an opening linewidth of about $100 \mu \mathrm{m}$. Figure 13 shows the Fresnel diffraction of the coded complex hologram at $z_{0}=4.5 \mathrm{~cm}$, which looks comparable with the results shown in Fig. 10(b). Figure 14 shows the pinhole hologram measured at $z_{0}=4.5 \mathrm{~cm}$, which is the impulse response of the system as we have placed a pinhole of diameter of $0.28 \mathrm{~mm}$ as the object being scanned. Figures 14(a) and 14(b) should be compared with the simulated results shown in Figs. 4(a) and 4(b), respectively. In the measured data in Fig. 14, we noticed there are fine fringes along the southwest and northeast corners of the ring. However, this aspect has not been noticed in the simulated results shown in Fig. 4 . We believe there are a couple of things that cause the discrepancies. First, there is aliasing associated with the simulated results in Fig. 4. Second, the pinhole used to measure the pinhole holograms is of some finite size, which renders the loss of resolution in the measurements. These also explain why the measured results shown in Fig. 12 do not coincide well with those shown in Fig. 6 . However, the measured result shown in Fig. 13 looks very similar to that of Fig. 10. In any case, in Fig. 15, we show the decoding of the complex coded hologram using the pinhole hologram shown in Fig. 14. We see a clear decoded image. To illustrate the $3 \overline{\mathrm{D}}$ complex coding capability, we code a $3 \mathrm{D}$ object in a single scanning. Now the "V" is located at $z_{0}=0$ and the "T" at $z_{0}=4.5 \mathrm{~cm}$. Thus, the object to be scanned contains a volume instead of a plane. The coded complex hologram of such a 3D object is shown in Fig. 16. To correctly decode the hologram, we also need to measure the pinhole hologram at $z_{0}=0$, which is shown in Fig. 17. Figure 18 shows the decoded image using the pinhole holograms shown in Figs. 14 and 17. We see that the "V" and "T" are correctly decoded at different planes. 


\section{Concluding Remarks}

We have proposed and demonstrated 3D complex coding and decoding with a CDG as the coding pupil in the context of OSH. Because the CDG is a purely phase grating, the decoding can be performed by using the same grating. To correctly decode the $3 \mathrm{D}$ image on a specific depth plane, we measured and used the pinhole holograms (the impulse responses) for decoding. This is advantageous because any imperfection in the optical system, such as aberration, can be alleviated in practice. In contrast to the previous intensity-based coding method [3], which can only code the image at the focal plane, the proposed method can be used for complex coding of a 3D object.

We have also pointed out that any pure phase patterns, such as a random phase diffuser, may also be used as a coding pupil for 3D coding and decoding. However, it is hard to fabricate or duplicate a random phase diffuser $[15,16]$. Also in practice, light diffraction from a random phase diffuser may be too weak to detect. Indeed, many proposed encryption/decryption processing techniques remain difficult to be implemented using optical techniques. Using a CDG is one of the easiest ways to realize such $3 \mathrm{D}$ complex coding optically. The grating provides light efficiency in the system in the form of one optical ring on the object being coded. In real-time applications, security can be enhanced if different holograms are coded using CDGs with various grating periods. This can be done, for example, by a phase-modulation spatial light modulator.

We gratefully acknowledge the support of the General Research Fund (112908) of the Research Grants Council in Hong Kong. The research is also partially supported by the National Science Council of Taiwan (NSCT) (98-2918-I-035-002 and 98-2221-E-035-006). Finally, Xin Zhou is grateful to the China Scholarship Council for supporting his research in the U.S.

\section{References}

1. A. Alfalou and C. Brosseau, "Optical image compression and encryption methods,” Adv. Opt. Photon. 1, 589-636 (2009).

2. T.-C. Poon, "Optical scanning holography-a review of recent progress,” J. Opt. Soc. Korea 13, 406-415 (2009).

3. K. B. Doh, K. Dobson, T.-C. Poon, and P. S. Chung, "Optical image coding with a circular Dammann grating," Appl. Opt. 48, 134-139 (2009).

4. P. Refrégiér and B. Javidi, "Optical image encryption based on input and Fourier plane random encoding," Opt. Lett. 20, 767-769 (1995).

5. T.-C. Poon, T. Kim, and K. Doh, "Optical scanning cryptography for secure wireless transmission," Appl. Opt. 42, 6496-6503 (2003).

6. T.-C. Poon, Optical Scanning Holography with MATLAB (Springer, 2007).

7. T.-C. Poon, "Scanning holography and two-dimensional image processing by acousto-optic two-pupil synthesis," J. Opt. Soc. Am. A 2, 521-527 (1985).

8. H. Kim, S.-W. Min, B. Lee, and T.-C. Poon, "Optical sectioning for optical scanning holography using phase-space filtering with Wigner distribution functions," Appl. Opt. 47, D164D175 (2008).

9. X. Zhang, E. Y. Lam, and T.-C. Poon, "Reconstruction of sectional images in holography using inverse imaging," Opt. Express 16, 17215-17226 (2008).

10. E. Y Lam, X. Zhang, H. Vo, T.-C. Poon, and G. Indebetouw, "Three-dimensional microscopy and sectional image reconstruction using optical scanning holography," Appl. Opt. 48, H113-H119 (2009).

11. X. Zhang and E. Y. Lam, "Edge-preserving sectional image reconstruction in optical scanning holography," J. Opt. Soc. Am. A 27, 1630-1637 (2010).

12. C. Zhou, J. Jia, and L. Liu, "Circular Dammann grating," Opt. Lett. 28, 2174-2176 (2003).

13. F. J. Wen and P. S. Chung, "Use of the circular Dammann grating in angle measurement," Appl. Opt. 47, 5197-5200 (2008).

14. F. J. Wen, Z. Chen, and P. S. Chung, "Area measurement at long-distance using a circular Dammann grating," Appl. Opt. 49, 648-652 (2010).

15. W. C. Su, C. C. Sun, Y. C. Chen, and Y. Ouyang, "Duplication of phase key for random-phase-encrypted volume holograms," Appl. Opt. 43, 1728-1733 (2004).

16. C.-C. Chang, J.-P. Liu, H.-Y. Lee, C.-Y. Lin, T.-C. Chang, and H.-F. Yau, "Decryption of a random-phase multiplexing recording system," Opt. Commun. 259, 78-81 (2006). 